\title{
Quantum Dots in Photonic Crystals : From Single photon sources to single photon nonlinear optics
}

\author{
Arka Majumdar, Andrei Faraon, Dirk Englund, Ilya Fushman, Jelena Vučković* \\ E.L. Ginzton Laboratory, Stanford University, Stanford, CA, 94305, USA
}

\begin{abstract}
A quantum dot strongly coupled to a photonic crystal resonator is used to investigate cavity quantum electrodynamies phonomena in solid stato physics. Nonlinear optical phonomena such as photon blockade and photon induced tummeling are observed in this system. The nonlinearity of this system is sensitive to intra-cavity photon numbers close to unity, and it has been used to demonstrate conditional phase shifts of $28^{\circ}$ at a single photou level and a second order auto-comelation of $g^{2}(0)=0.9$ in the photon blockade regime.
\end{abstract}

Keywords: quantum information, photonic crystal, quantum dot, single photon source

\section{INTRODUCTION}

Advancements in semiconductor fabrication techology and cavity quantum electrodynamics (QED) have lod to a vast research effort in the field of cavity QED in scmiconductor based cavity system. Researchers recently demonstrated strong light-matter interaction in different semiconductor cavities ${ }^{123}$ including micro-discs, microposts and photonic crystal cavities. In our research, we work with photonic crystal cavities.

Photonic crystals (PC) are optical media where the refractive index changes in a periodic fashion. They can be used to guide and confine light by distributed Bragg reflection (DBR). By inserting some defects in that periodic structure (e.g., removing some holes) one can make a structure (Figure 1) where light gets confined in plane by DBR and out-of-plane by total internal reflection (TIR). For the experiments described in this paper we use a linear throe hole defoct cavity (L3) constructed by omitting three holes in the photonic crystal lattice as shown in Fig. 1. Quantum dots (QDs) are semiconductor hetero-structures that provide three dimensional confinement for electrons and holes. This confinement gives rise to a delta-function like density of states and makes the QD manifest atom-like properties. Hence it is sometimes regarded as an artificial atom. ${ }^{5}$ When the QD is spectrally and spatially matched with the cavity, the QD and the cavity can coherently exchange encrgy between themselves and thus forms a system to probe light matter interaction in the regime of cavity QED.

In cavity quantum electrodynamics (QED), there are two distinct regimes: weak coupling regime where the coherent coupling rate $(g)$ between the cavity and the atom is less than the decay rate from the cavity $(\kappa)$ and the QD $(\gamma)$; and the strong coupling regime where $g$ is larger than $k$ and $\gamma$. The most interesting cavity QED effects occur when the QD is strongly coupled to the cavity. In this regime, the energy of the system coherently oscillates between the QD and the resonator, an effect known as Rabi oscillations. In semiconductor micro-cavity systems onc can achieve another regime (called Purcell enhanced regime) where $g\left\langle\kappa\right.$ but $g^{2} / \kappa \gamma>>1$. Cavity QED effects as spontaneous emission rate enhancement and dipole induced transparcncy can be observed in this regime. ${ }^{6}$

One major problem of coupling a QD to cavity is the inhomogeneous broadening of the QD. To reach strong: coupling, one needs to couple the QD to the cavity both spectrally as well as spatially. We have developed local QD and cavity tuming techniques, ${ }^{78}$ to overcome the challenge of spectral matching.

In this paper, we describe our recent effort to probe light-matter interaction in a QD coupled to a PC cavity. The paper is organized as follows: in section 2 we describe our system parameters, in section 3 we describe the coherent probing of cavity-QD system. In section 4 and 5 we describe two potential applications of this type of system: controlled phase shifts at single photon level and generation of non-classical light by photon blockade and photon-induced tumneling. In section 6 we comment on the future directions of this work and in section 7 we conclucle the paper.

*To whom correspondence should be addressed. Email: jela@stanford.edu 
d

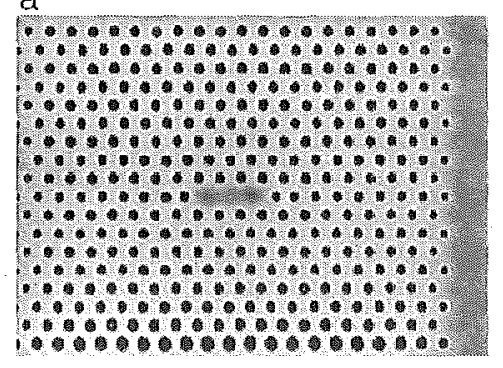

b
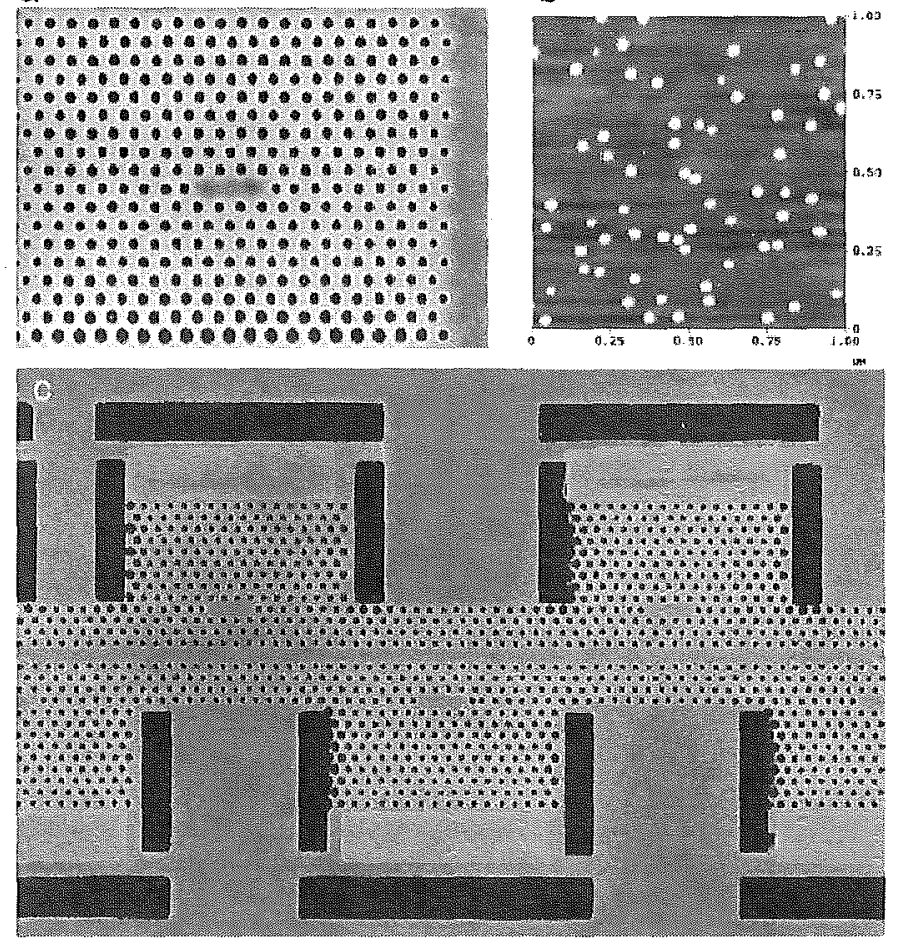

Figure 1. (a) Scanning electron microscope image of the fabricated structure showing the photonic crystal structure and the cavity, (b) A chip containing Quantum Dots, (c) Scanning electron micro-graph of a bypothetical integrated nano-photonic structure made of photonic crystal cavitics and wave-gudies.

\section{SYSTEM CHARACTERIZATION}

Our optical system consists of a photonic crystal cavity fabricated in a $160 \mathrm{~nm}$ thick GaAs membrano by a combination of electron bean lithography and dry/wet etching steps, as discussed in Ref. ${ }^{1}$ The membrane contains a central layer of self-assembled InGaAs QDs with an estimated density of $50 / \mu m^{2}$. The system is first characterized by the photolmminescence (PL) observed from the cavity/QD, when it is pumped above-band by a continuous wave (CW) Ti-Sapphire laser. The frequency of the laser is optimized by observing the dot and cavity spectrum in the spectrometer. We tune the system from $4 K$ to $50 K$ and observed anti-crossing as shown in Figme 2. The anti-crossing is a signature of the strong coupling between cavity and the QD. In this particular instant, the system paraneters are $g / 2 \pi=8 \mathrm{GHz}, \kappa / 2 \pi=16 \mathrm{GHz}, \gamma=\kappa / 100$; where $g$ is the atom-cavity coupling strength; $k$ is the cavity decay rate and is given by $\omega_{c} / Q\left(Q\right.$ is the Q-factor of the cavity and $\omega_{c}$ is the resonance energy of the cavity); $\gamma$ is the QD spontancous emission rate. 'To find other higher order electronic states of the QD we do power series measurement. The cavity and single exciton lines follow a linear relation with the laser power, whereas the bi-exciton follows a square law.

\section{CONTROLLING CAVITY REFLECTIVITY BY SINGLE QUANTUM DOT : GIANT OPTICAL NON-LINEARITY}

Once the system is characterized in PL and a strongly coupled dot is found, the system is probed resonantly. Here we resonantly excite the system by a laser and measure the transmission of the laser through the coupled cavity/QD system. To get rid of the excitation background we used cross-polarized setup, where we excite the system by a vertically polarized light and observe the scattered horizontal light from the cavity. The reflectivity signal from a cavity is modified by the presence of a strongly coupled dot. During our initial experiments the laser 

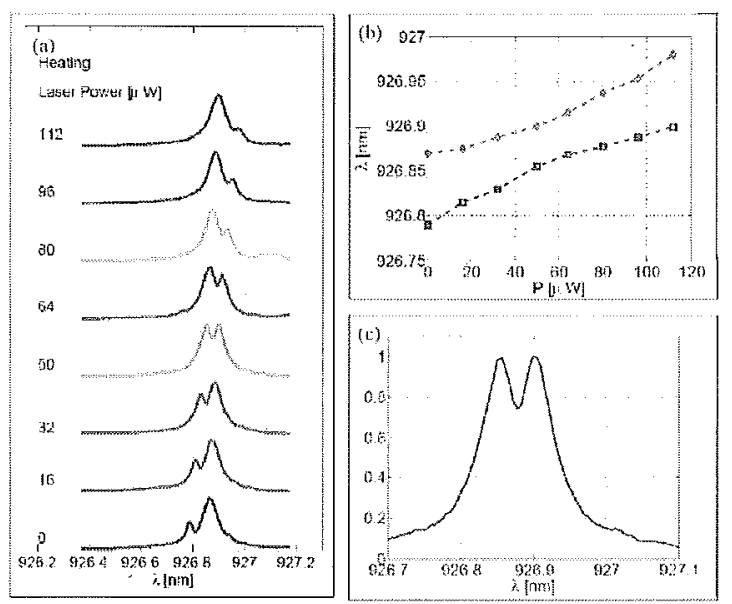

Figure 2. (a)The anti-crossing of the cavity and the QD. We use laser-heating to tume the QD and the cavity. As can be seen from the figure there is no crossing of cavity and the Quantum dot. (b) The cavity and the QD resonance frequeneies are plotted as a function of heating laser power. (c) The normal mode spliting when the QD and the cavity are in resonance.

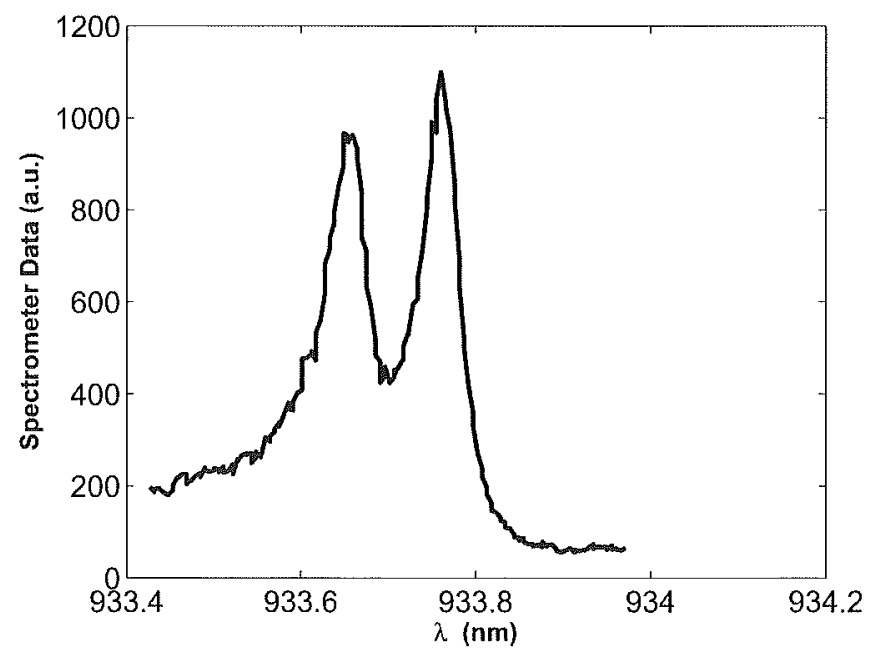

Figure 3 . The cavity is coherently probed by the laser. The Strongly Coupled dot (in resonance with the cavity) modifics the enpty cavity spectrum. This reflectivity signal is taken from a different dot than shown in Figure 2.

wavelength was difficult to tune continuously due to the mode-hopping of the laser. The scanning was achieved by keeping the laser wavelength constant and changing the cavity and the QD resonances by temperature tuning. ${ }^{1}$ In our more recent experiments the reflectivity measurement is done by scamning a NewFocus 6319 tunable laser with linewidth $<300 k H z$. A spectrum obtained by this method is shown in Fig. 3. One can observe that the QD induces a split of the cavity spectrum that has a Lorentzian shape in the uncoupled case. We also showed that the dip in reflectivity can be reduced by saturating the dot ${ }^{1}$ with a very few intra-cavity photon and thus we showed a giant optical nonlinearity in a strongly coupled cavity/QD system (Figure 4 ). Rererence ${ }^{4}$ gives a. good theoretical treatment of this nonlinearity. 


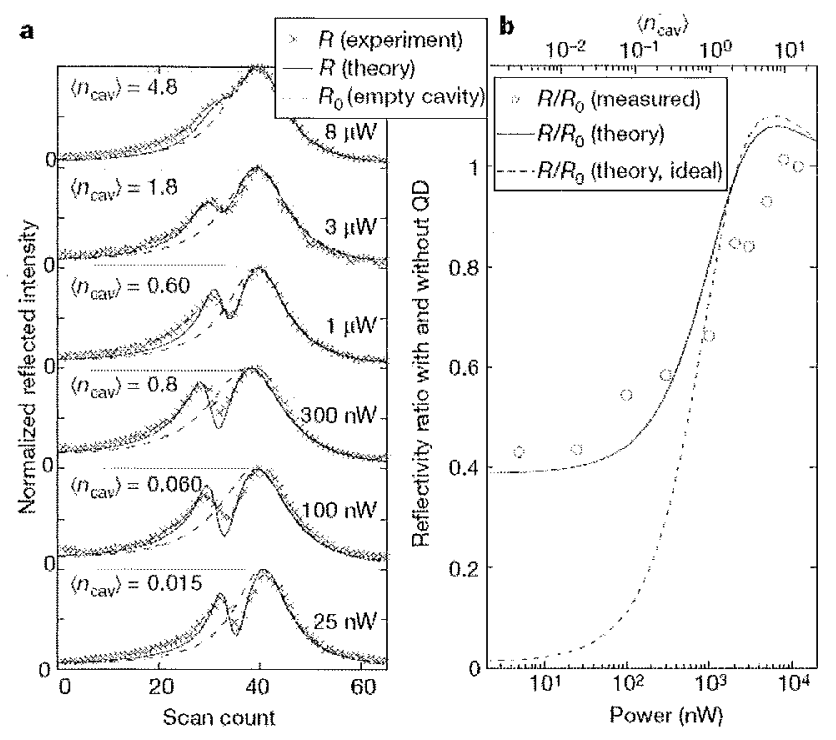

Figure 4. (a) Reflectivity scans at increasing probe power (meastred before the objective), ranging from low-excitation to saturation regimes. The measured reflectivity is fitted by a numerical solution to the full master equation, (b) Reflectivity ratio of a cavity with dot and an empty cavity as a function of power of the exciting laser. Saturation is observed at higher photon numbers. The difference between theory and ideal theory is caused by thermal fluctuation.

\section{CONTROLLED PHASE SHIFTS WITH A SINGLE QUANTUM DOT AND A SINGLE PHOTON}

Quantum circuit model shows that a controlled phase flip gate along with single qubit operations constitute a universal set of gates for Quantum Computation. ${ }^{10}$ We showed that a PhC cavity coupled with a QD has the potential to implement a controlled phase flip gate ${ }^{11}$ as depending on the photon numbers inside the cavity, the light gets a non-linear phase shift. This nonlinearity is again due to the saturation of the QD (when both signal and control beam are of same wavelength). The maximum phase difference obtained between single and two photons is $13^{\circ}$ at an intra-cavity photon number of 0.1.11 Hence we can achieve non-linear optics in single photon level. Cascading several of these systems, we can achieve a controlled phase flip gate which provides a $\pi$ phase-shift between input and output light depending on photon numbers. Figure 5 shows the signal phase difference as a function of incoming photon. By using two different frequency photons, we can achieve more phase shift $\left(28^{\circ}\right)$. This is caused by the saturation of the QD and a frequency shift of the QD due to the ac: Stark effect, which can be used to realize large phase shifts. Figure 6 shows the signal phase difference when two different frequency light is used for signal and control.

\section{NON-CLASSICAL STATE GENERATION BY PHOTON BLOCKADE}

This strongly coupled system can also be potentially used to probe the photon blockade similar to those in atomic physics experiments. ${ }^{12}$ Here we excite the system with a pulsed laser when the QD and the cavity are resonant and find the second order autocorrelation of the cmitted light by means of a HBT setup. For classical light (where the photons follow a poissonian distribution) the second order autocorrelation $g^{2}(0)$ is 1 . For a single photon $g^{2}(0)=0$. We observed anti-bunching of light $\left(g^{2}(0)<1\right)$ when the laser is resonant with the polaritons (the eigen-states of the QD-cavity system). But during our experiment, we found another feature (which is previously known theoretically but not demonstrated experimentally) of bunching $\left(g^{2}(0)>1\right)$ of light when the laser is resonant with the cmpty cavity resonance. We call it photon induced tunneling. ${ }^{13}$ Figure 7 shows the second order autocorrelation with different exciting frequencies of the laser. 


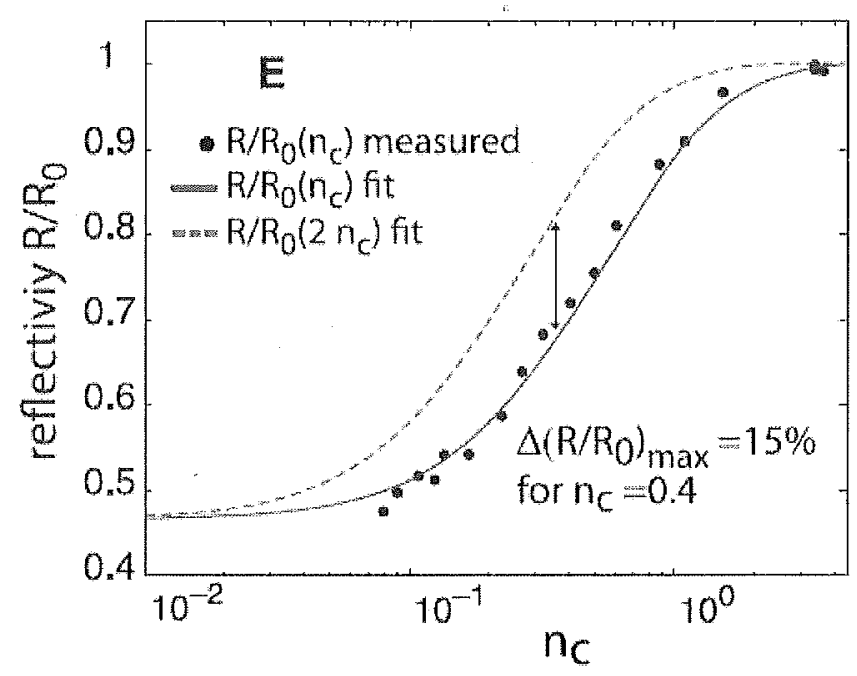

Figure 5. The signal Phase derived from the experiment as a function of the intra-cavity photon. The blue line is the theoretical plot which matches quite well with the experimentally obtained data-points. The red line is extrapolated from the blue line and shows the expected phase shift when the intra-cavity photon number doubles. 'The difference between two lines is the non-linear phase shift.

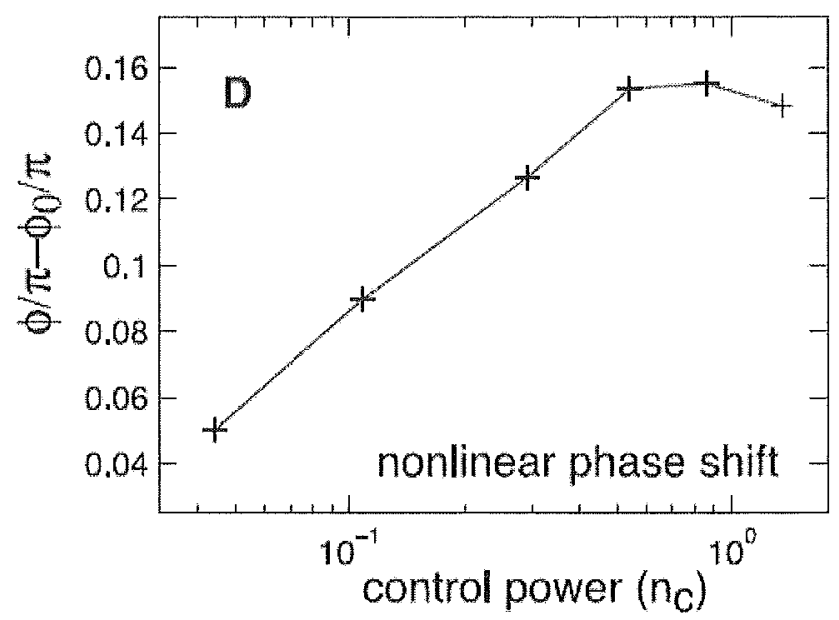

Figure 6. Difference between the phase shift of the signal beam when the control beam is on and when it is off as a function of intra-cavity photon number. 


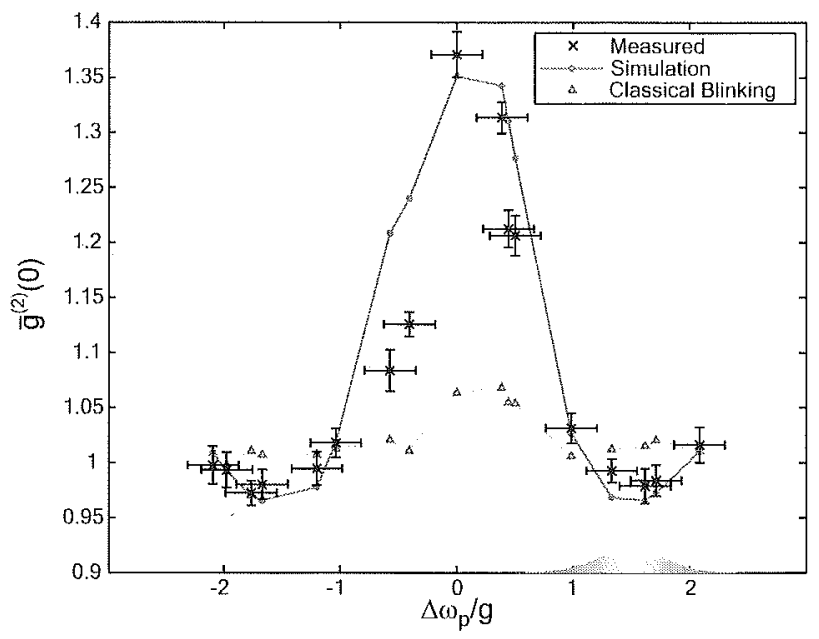

Figure 7. The second-order autocorrelation with the detuming $\left(\Delta \omega_{p}=\omega_{p}-\omega_{c}\right)$ of the laser frequency $\left(\omega_{p}\right)$ with the cavity resomance frequency $\left(\omega_{c}\right)$. We can see that at $\Delta \omega_{p} / g= \pm 1.5$ we get anti-bunching. The deviation from intuitive value of $\Delta \omega_{p} / g= \pm 1$ is due to the finite line width of the cavity and the Quantum Dot. Also at $\Delta \omega_{p}=0$ we see bunching.

The minimum $g^{2}(0)$ obtained from our experiment is 0.9 . This value can be potentially improved by shaping the exciting laser pulse in spatial and time domain and thus improving the coupling of the first photon to the cavity. Cascading two cavities with QD is also a solution, but in present technology it is difficult to achieve due to very little control of positioning the QDs in desired places accurately.

\section{FUTURE DIRECTIONS}

After this initial demonstration of cavity/QD system as a potential candidate for Quantum information science, the next step will be to create entanglement between two distant QD/cavity system. For this, one needs multilevel system in a QD. There are proposals for using exciton-biexciton system to achieve that. ${ }^{14}$ Also, the effect of two modes are interesting to look into, as already done in a micro-disk and atomic system. ${ }^{15}$ Apart from these, we are also working on making an integrated nano-photonic structure (for Quantum Information Processing), some of which have been demonstrated already. ${ }^{16}$

\section{CONCLUSION}

We have performed cavity QED experiments in Photonic crystal cavity and QD system. The preliminary results show that this system is well-suitod for quantum information scicnce.

\section{REFERENCES}

1. Dirk Englund, Andrei Faraon, Ilya Fushman, Nick Stoltz, Pierre Petroff, Jelena Vuckovic, "Controlling cavity reflectivity with a single quantum dot," Nature, Vol 450, 6 December 2007, pp.-857 - 861.

2. K. Hemessy, A. Badolato, M. Winger, D. Gerace, M. Atatüre, S. Gulde, S. Fält, E. L. Hu ,A. Imamoglu, Quantum nature of a strongly coupled single quantum dotcavity systen, Nature 445, 896-899 (22 February 2007).

3. Kartik Srinivasan, Oskar Painter, "Linear and nonlinear optical spectroscopy of a strongly coupled microdiskquantum dot system," Nature, Vol 450, 6 December 2007. pp.-862 - 865. 
4. Alexia Auffèves-Garnier, Christoph Simon, Jean-Michel Gèrard, and Jean-Philippe Poizat,"Griant optical nonlinearity induced by a single two-level system interacting with a cavity in the Purcell regime," Phys. Rev. A 75, 053823 (2007).

5. S. W. Koch, M. Kira, G. Khitrova and H. M. Gibbs, "Semiconductor excitons in new light," Nature Materials $5,523-531(2006)$.

6. Edo Waks and Jelena Vuckovic, "Dipole Induced Transparcncy in Drop-Filter Cavity-Waveguide Systcms,"PRL 96, 153601 (2006).

7. Andrei Faraon, Dirk Englund, Ilya Fushman, Nick Stoltz, Pierre Petroff, Jelena Vuckovic, "Local Quantum Dot Tuning on Photonic Crystal Chips," Applied Physics Letters, Vol 90, article 213110 (2007).

8. Andrei Faraon, Dirk Englund, Douglas Bulla, Barry Luther-Davies, Benjamin J. Eggleton, Nick Stoltz, Picre Petroff, Jclena Vuckovic, "Local Tuning of Photonic Crystal Cavities Using Chalcogenide Glasses," Applied Physics Letters, Vol 92, article 043123 (2008).

9. Antonio Badolato, Kevin Hennessy, Mete Atatüre, Jan Dreiser, Evelyn Hu, Pierre M. Petroff, Atac Imamoglu, "Deterministic Coupling of Single Quantum Dots to Single Nanocavity Modes," Science 20 May 2005 Vol. 308. no. 5725, pp. $1.158-1161$.

10. Michael Nielsen, Isaac L. Chuang, "Quantum Computation and Quantum Information," Cambridge University Press.

11. Ilya Fushman, Dirk Englund, Andrei Faraon, Nick Stoltz, Pierre Petroff, Jelena Vuckovic, "Controlled Phase Shifts with a Single Quantum Dot," Science, vol. 320, number 5877, pp. 769 - 772 (2008).

12. K. M. Bimbaum, A. Boca, R. Miller, A. D. Boozer, T. E. Northup, H. J. Kimble, "Photon blockade in an optical cavity with one trapped atom," Nature 436, 87 - 90 (7 July 2005).

13. Andrei Faraon, Ilya Fushman, Dirk Englund, Nick Stoltz, Pierre Petroff, Jelena Vuckovic, "Coherent generation of nonclassical light on a chip via photon-induced tumneling and blockade," Nature Physics, 4, 859-863, (2008).

14. Deepak Sridharan and Edo Waks, "Generating entanglement between quantum dots with different resonant frequencies based on dipole induced transparency,"Phys. Rev. A 78, 052321 (2008).

15. Barak Dayan, A. S. Parkins, Takao Aoki, E. P. Ostby, K. J. Vahala, H. J. Kimble," Photon Turnstile Dynamically Regulated by One Atom," Science, 22 February 2008, Vol. 319 no. 5866, pp. 1062 - 1065.

16. Andrei Faraon, Ilya Fushman, Dirk Englund, Nick Stoltz, Picrre Petroff, Jelena Vuckovic, "Dipole induced transparency in waveguide coupled photonic crystal cavities," Optics Express, Vol 16, pp 12154-12162 (2008). 


\section{PROCEEDINGS OF SPIE}

\section{Physics and Simulation of Optoelectronic Devices XVII}

Marek Osiński

Bernd Witzigmann

Fritz Henneberger

Yasuhiko Arakawa

Editors

26-29 January 2009

San Jose, California, United States

Sponsored and Published by

SPIE 